Article

\title{
Sport-Specific Habitual Adaptations in Neck Kinesthetic Functions Are Related to Balance Controlling Mechanisms
}

\author{
Ziva Majcen Rosker ${ }^{1, *(1)}$ and Miha Vodicar ${ }^{2}$ \\ 1 Faculty of Sport, University of Ljubljana, 1000 Ljubljana, Slovenia \\ 2 Department of Orthopedic Surgery, University of Ljubljana, 1000 Ljubljana, Slovenia; miha.vodicar@kclj.si \\ * Correspondence: ziva.majcen-rosker@fsp.uni-lj.si
}

Received: 26 November 2020; Accepted: 14 December 2020; Published: 16 December 2020

\begin{abstract}
Whilst the importance of trunk, lower and upper extremities for the efficiency of maintaining body sway is well documented, the effects of cervical spine function have been seldom investigated. Afferent information from high density proprioceptors located in the cervical spine can alter postural balance, however the effects of sport's specific habitual adaptation on balance performance have not yet been investigated. Twenty-seven taekwondo fighters and thirty controls performed unilateral balance tasks while facing forward and during neck torsion balance test while standing on the force plate. Neck kinesthesia was measured with the Head-to-Neutral Relocation test and the Butterfly test with motion-inertial unit. Differences between balance tasks were analyzed using two-way ANOVA. Additionally, correlations between body sway parameters and neck kinesthesia were studied using Pearson correlation coefficient. No differences were found between forward facing and neck torsion balance tasks in taekwondo fighters. However, correlations were found between balance on the non-preferred leg and neck kinesthesia. On the contrary, healthy individuals presented with statistically significant differences between both balance tasks and correlations between balance and neck kinesthesia. Taekwondo fighters seem to present with habitual adaptations in balance control, that differ from non-trained individuals.
\end{abstract}

Keywords: neck proprioception; body sway; postural balance

\section{Introduction}

Taekwondo fighters require sufficient postural stability to better maintain tactical superiority over an opponent [1]. Up-to-date research is focused towards investigating postural balance during bipedal and unipedal stances while facing forward [2]. However, taekwondo-specific movements during a fight require them to adapt a guard-specific posture which involves slight trunk rotation with increased neck torsion towards the opponent. The aforementioned posture requires them to frequently use one leg as "standing" leg, while the other one as "kicking" leg, commonly referred to as preferred and non-preferred leg [3]. This could cause important asymmetries in unilateral stances and could be even more pronounced in guard-specific posture with the neck rotated towards the opponent. Asymmetries in unilateral stances caused by changes in different neuromuscular functions could be accompanied by important adaptations in proprioceptive system [4] with afferent input causing additional alterations in efferent drive to the peripheral effectors. While efferent input adjusts for final motor responses, preprograming of movement is dependent on the variety of sensory sources. Literature reports on importance of integrating different sensory sources for postural sway control, with articles addressing primarily the importance of ankle and plantar coetaneous receptors as well as more proximal sensory drive [5]. According to Boyd-Clark et al. [6], the cervical spine holds abundance 
of proprioceptors within the structures of the spine and muscles with muscle spindles especially sensitive to head yaw rotation [7]. Basic motor control studies suggest that cervical spine is highly susceptible to mechanical stimuli and habitual adaptations due to prolonged time spent in altered posture which further influences body awareness and spatial orientation [8].

Since guard-specific posture in taekwondo holds unilateral rotation of the cervical spine, long term adaptations in kinesthetic awareness from the neck could influence balance performance. In addition, cervical spine governs head movements and has a direct neurophysiological connection to the vestibular and visual system, therefore adaptations in neck proprioceptive system could influence vestibular and visual system and consequently further influence balance performance [9]. Based on these suggestions, it would be of importance to consider investigating balance performance during the neck torsion balance test, since literature suggests that the remaining test can screen for those with cervical spine deficits. According to Williams et al. [10] Neck torsion balance test importantly differentiates between those with an altered afferent input from cervical proprioceptors and those without, but comparisons were only done between whiplash patients and healthy individuals. The results of their study suggest that individuals with cervical spine deficits present with poorer balance when neck is in torsion and then facing forward. Since altered kinesthetic awareness can be seen in healthy athletes [11], especially those with asymmetrical proprioceptive deficits, afferent input from cervical spine during the neck torsion balance test could influence balance performance.

The main goal of our study was to investigate whether neck kinesthetic functions corelate to balance performance in different stances and whether balance deficits are more pronounced in guard-specific posture. The remaining relationship could give further insight into the balance-controlling mechanisms in taekwondo fighters.

\section{Materials and Methods}

\subsection{Participants}

Fifty-seven participants were enrolled in the study of which 30 were non-trained male individuals (average age $20.4 \pm 5.6$ years, height $1.79 \pm 0.04 \mathrm{~m}$ and body mass $84.2 \pm 7.4 \mathrm{~kg}$ ) and 27 male taekwondo fighters from the junior and senior category competing at national and international level (average age $18.3 \pm 3.3$ years, height $1.79 \pm 0.08 \mathrm{~m}$, body mass $70.3 \pm 15.1 \mathrm{~kg}$ and average training experiences $14 \pm 5.1$ years). Participants were enrolled in the study if they did not report any of the following complaints: head and neck pain, neck pain related symptoms, lower limb or trunk injuries in the last six months and neurological or vestibular disorders. All individuals were not allowed to drink any alcohol or take medication for at least $24 \mathrm{~h}$ prior to testing. All participants were required to read and sign an informed consent form. The study was approved by the National Committee for Medical Ethics (number 0120-47/2020/6, 18 February 2020) and performed according to the Declaration of Helsinki.

\subsection{Measurement Procedures}

Body sway, cervical spine kinesthetic awareness (Butterfly test) and head and neck position sense (Head-to-Neutral) tests were performed. Postural balance was assessed using the following tasks: single leg stance for both legs, single leg stance on the left leg while the neck was rotated by $90^{\circ}$ to the right and single leg stance on the right leg with neck rotated $90^{\circ}$ to the left (Figure 1). All balance tasks were performed with opened eyes. Participants were instructed to remain focused on a dot on the wall which was placed at a $2 \mathrm{~m}$ distance. They were required to place their hands on the hips and to keep their legs straight, but not hyperextended. During all tasks, they had to stand as still as possible for three times $30 \mathrm{~s}$ with $60 \mathrm{~s}$ rest intervals. All balance tests were performed in a random order. Center of pressure (CoP) was measured while standing on the force plate (9260AA, Kistler Instruments AG, Winterthur, Switzerland) and analyzed with Kistler MARS (Kistler, Winterthur, Switzerland). 
A

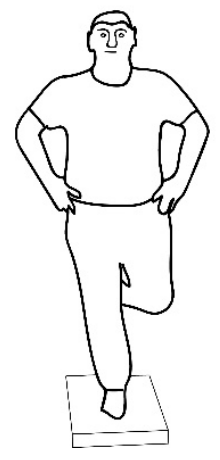

B

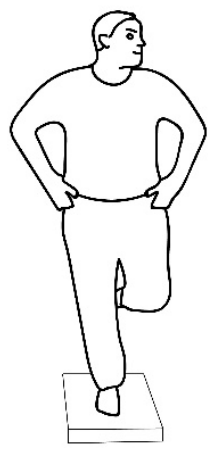

Figure 1. Stances during balance assessment: forward facing (A) and neck rotated $90^{\circ}(\mathbf{B})$ balance tasks.

Neck kinesthesia was tested using a Butterfly test (Butterfly Method, NeckCare ehf., Reykjavik, Iceland). The method was described in detail elsewhere [12]. Head and neck movements were measured with a NeckCare sensor (NeckGear, NeckCare ehf, Reykjavik, Iceland) positioned on the participants' heads. During the test, participants were sitting upright and were instructed to track a target moving unpredictably on the computer screen as accurately as possible. Movement trajectories of the target (easy, medium and difficult) and test duration were predefined by the NeckCare software (Butterfly Method, NeckCare ehf., Reykjavik, Iceland).

Proprioception, more specifically position sense, was measured with a Head-to-Neutral Relocation test using NeckGear sensor. Participants sat blindfolded. Before each trial their head and neck were placed in a neutral position, which served as a reference posture. Participants had to perform slow head movements in the transverse plane around the vertical axis (i.e., rotations) and in the sagittal plane around the transverse axis (i.e., flexion and extension) before returning back to the neutral position. All trials were repeated six times consecutively.

\subsection{Data Analysis}

CoP movement was acquired and analyzed using LabView based software (ARS Force Plate, S2P, Ljubljana. Slovenia). Signals were sampled at $1000 \mathrm{~Hz}$ and filtered $(0.04-10 \mathrm{~Hz}$ band-pass, Butterworth zero-lag fourth-order). The following parameters describing $\mathrm{CoP}$ movement were calculated; (i) average velocity of CoP movement (Vtot), (ii, iii) velocity of CoP movement in medial-lateral (ML) (Vml) and anterior-posterior (AP) (Vap) direction, (iv, v) average amplitude of CoP movement in ML (Aml) and AP (Aap) direction, (vi, vii)mean frequency of changes in CoP movement direction during ML (Fml) and in AP (Fap) postural sway.

Head and neck movements during the Butterfly test were analyzed using the NeckCare software. Parameters of (i) mean and standard deviation of the time spent on target during each trial expressed in seconds (time-on-target), (ii) time head and neck spent behind the target expressed as percentage of total time (undershoot), (iii) time head and neck spent in front of the target expressed as percentage of total time (overshoot) and (iv) average mismatch between the target and the head cursor in millimeters (amplitude accuracy) were used for further analysis.

NeckCare software was used to analyze relocation accuracy in the Head-to-Neutral position sense. Parameters such as (i) mean of the absolute total deviation from the reference position over the six trials for each measured direction (absolute error) and (ii) average magnitude of under- and over-estimation of target position (constant error) were further analyzed.

\subsection{Statistical Analysis}

Statistical analysis was performed in SPSS (SPSS 23.0 software, SPSS Inc., Chicago, IL, USA). Normality of distribution was analyzed using Kolmogorov-Smirnov test. Differences between stance 
(facing forward and head rotation (2)) and leg (preferred and non-preferred (2)) were analyzed using two-way repeated measures analysis of variance. Effect size was calculated using the partial eta square $\left(\eta_{p}{ }^{2}\right)$, and was treated as $\eta_{p}{ }^{2}>0.01$-small, $0.06<\eta_{p}{ }^{2}<0.14$-medium and high when $\eta_{p}{ }^{2}$ was higher than 0.14 [13]. Post-hoc two tailed T-test for dependent samples was used for analyzing differences between individual factors. The effect size was calculated using Cohens $\mathrm{d}(\mathrm{d})$. Pearson correlation coefficient ( $\mathrm{r}$ ) was used to test for correlations between parameters of balance with Head-to-Neural Relocation test and the Butterfly test. The $\mathrm{r}$ was treated as no correlation for $\mathrm{r}<0.3$, small correlation for $0.3<\mathrm{r}<0.5$, medium correlation for $0.5<\mathrm{r}<0.7$ and high correlation for $\mathrm{r}>0.7$ [14]. The level of statistical significance $(p)$ was set at $p<0.05$. All $p$ values in correlation tests were adjusted for multiple comparisons according to the Benjamini and Hochberg procedure [15].

\section{Results}

The results of two-way repeated measures analysis of variance investigating differences between balance tasks are presented in Table 1. No significant differences between two different stances and legs were found in the taekwondo group. In the control group, statistically significant differences were found between the stances for the parameter sway path and parameter average amplitude in the anterior-posterior direction. Post-hoc analysis revealed significant differences in sway path in the anterior-posterior direction for the forward facing and trunk rotated to the dominant side $(t=5.627$, $p=0.042, \mathrm{~d}=2.412$ ) as well as for the forward facing and trunk rotated to the non-dominant side $(\mathrm{t}=4.948, p=0.048, \mathrm{~d}=1.718)$ in the control group.

Table 1. Differences between balance tasks.

\begin{tabular}{|c|c|c|c|c|c|c|c|}
\hline \multirow{2}{*}{\multicolumn{2}{|c|}{ Group/Parameter }} & \multicolumn{3}{|c|}{ Stance } & \multicolumn{3}{|c|}{ Leg } \\
\hline & & $\mathbf{F}$ & $\mathbf{P}$ & $\mu^{2}$ & F & $p$ & $\mu^{2}$ \\
\hline \multirow{7}{*}{ 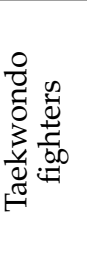 } & Sptot & 0.400 & 0.534 & 0.018 & 0.006 & 0.941 & 0.000 \\
\hline & Spap & 0.766 & 0.391 & 0.034 & 0.177 & 0.678 & 0.008 \\
\hline & Spml & 0.065 & 0.801 & 0.003 & 0.588 & 0.451 & 0.026 \\
\hline & Aap & 2.302 & 0.143 & 0.095 & 0.038 & 0.847 & 0.002 \\
\hline & Aml & 0.203 & 0.657 & 0.009 & 2.021 & 0.169 & 0.084 \\
\hline & Fap & 1.825 & 0.190 & 0.077 & 0.308 & 0.585 & 0.014 \\
\hline & Fml & 0.105 & 0.749 & 0.005 & 2.870 & 0.104 & 0.115 \\
\hline \multirow{7}{*}{ 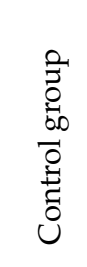 } & Sptot & 2.452 & 0.042 & 0.150 & 1.057 & 0.318 & 0.006 \\
\hline & Spap & 4.474 & 0.021 & 0.304 & 1.284 & 0.231 & 0.020 \\
\hline & Spml & 1.117 & 0.081 & 0.011 & 0.841 & 0.422 & 0.001 \\
\hline & Aap & 3.264 & 0.019 & 0.270 & 0.873 & 0.405 & 0.004 \\
\hline & Aml & 0.945 & 0.161 & 0.091 & -0.247 & 0.811 & 0.015 \\
\hline & Fap & 0.737 & 0.284 & 0.067 & -0.224 & 0.828 & 0.020 \\
\hline & Fml & 0.699 & 0.398 & 0.102 & 1.429 & 0.187 & 0.004 \\
\hline
\end{tabular}

SPtot-distance by the center of pressure movement; SPap-distance by the center of pressure movement in anterior-posterior direction; SPml-distance by the center of pressure movement in medial-lateral direction; Aap-average amplitude of center of pressure movement in anterior-posterior direction; Aml—average amplitude of center of pressure movement in medial-lateral direction; Fap-average frequency of center of pressure direction changes in anterior-posterior direction; Fml-average frequency of center of pressure direction changes in medial-lateral direction; $\mathrm{F}-\mathrm{f}$ statistic, $p$-statistical significance; $\mu^{2}$-partial eta square.

The results of the correlation analysis between Head-to-Neutral proprioception test, Butterfly test and body sway parameters are presented in Table 2. The taekwondo group showed statistically significant medium sized correlations between Head-to-Neutral from preferred rotation direction and sway path in the anterior-posterior direction, high correlations between Head-to-Neutral from the forward direction and frequency of body sway in the medial-lateral direction. In addition, medium-to-high correlations were observed between overshoot and all the sway path and average amplitude body sway parameters. These correlations were absent in the head rotation to the non-preferred side balance tasks, except for the high correlations between the undershoot and the medial-lateral sway path, total sway path and average amplitude in both anterior-posterior and 
medial-lateral direction. For the control group, all body sway parameters during forward facing balance task were highly correlated to the time on target during the Butterfly test.

Table 2. Correlations between neck kinesthesia and postural balance.

\begin{tabular}{|c|c|c|c|c|c|c|c|}
\hline \multirow{3}{*}{\multicolumn{2}{|c|}{ Group/Parameter }} & \multicolumn{5}{|c|}{ Non-Dominant Leg } & \multirow{3}{*}{$\begin{array}{c}\text { Dominant Leg } \\
\text { Control Group } \\
\text { ToTd }\end{array}$} \\
\hline & & \multicolumn{4}{|c|}{ Taekwondo Fighters } & \multirow{2}{*}{$\begin{array}{c}\text { Control Group } \\
\text { ToTd }\end{array}$} & \\
\hline & & Um & Od & Hrv & Hfc & & \\
\hline \multirow{7}{*}{ 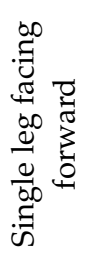 } & SPtot & 0.417 & $0.772^{* *}$ & -0.618 & -0.040 & $0.846^{*}$ & $0.832 *$ \\
\hline & SPap & 0.558 & $0.667 *$ & -0.678 * & -0.186 & $0.813 *$ & 0.789 \\
\hline & SPml & 0.204 & $0.830^{* *}$ & -0.498 & 0.106 & 0.860 * & 0.795 \\
\hline & Aap & 0.624 & $0.635^{*}$ & -0.614 & 0.149 & 0.889 * & 0.804 * \\
\hline & $\mathrm{Aml}$ & -0.004 & 0.641 * & -0.200 & 0.553 & 0.914 * & 0.750 \\
\hline & Fap & -0.389 & -0.320 & 0.232 & -0.685 & -0.669 & -0.597 \\
\hline & Fml & 0.190 & -0.081 & -0.133 & $-0.812 * *$ & $-0.820 *$ & -0.597 \\
\hline \multirow{7}{*}{ 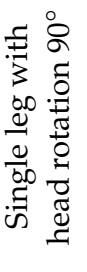 } & SPtot & $0.745^{*}$ & 0.504 & -0.698 & -0.073 & 0.787 & $0.918 *$ \\
\hline & SPap & $0.723 *$ & 0.541 & -0.695 & -0.024 & 0.779 & $0.944 *$ \\
\hline & SPml & 0.762 & 0.469 & -0.702 & -0.107 & 0.744 & 0.773 \\
\hline & Aap & $0.800 *$ & 0.614 & -0.689 & -0.021 & 0.786 & 0.945 * \\
\hline & $\mathrm{Aml}$ & 0.854 * & 0.507 & -0.735 & -0.109 & 0.726 & 0.861 \\
\hline & Fap & -0.609 & -0.622 & 0.461 & -0.094 & -0.600 & -0.557 \\
\hline & Fml & -0.646 & -0.426 & 0.596 & 0.008 & -0.477 & -0.621 \\
\hline
\end{tabular}

Parameters for which no significant correlations were found were excluded from the table. The following parameters are presented: Hrv—variable error for the Head-to-Neutral Relocation test from right; Hfc-constant error for the Head-to-Neutral Relocation test from flexion; Od-overshoot standard deviation of the Butterfly test; Um-undershoot standard deviation of the Butterfly test; SPtot—distance by the center of pressure movement; SPap-distance by the center of pressure movement in anterior-posterior direction; SPml-distance by the center of pressure movement in medial-lateral direction; Aap-average amplitude of center of pressure movement in anterior-posterior direction; Aml-average amplitude of center of pressure movement in medial-lateral direction; Fap-average frequency of center of pressure direction changes in anterior-posterior direction; Fml-average frequency of center of pressure direction changes in medial-lateral direction; numbers represent R; ${ }^{*} p<0.005 ;{ }^{* *} p<0.001$.

For the preferred leg, only the control group presented statistically significant correlations between time on target parameter and individual sway path and average amplitude in the anterior-posterior direction in both balance tasks (Table 2).

\section{Discussion}

Habitual adaptations due to a sport's specific posture can lead to changes in different sensory-motor functions. Taekwondo fighters continuously place their head and neck in a torsioned position, which can cause asymmetries in the neck. These can lead to altered proprioceptive feedback which is suggested to influence balance performance [16]. The first aim of this study was to investigate whether neck kinesthetic functions corelate to balance performance in taekwondo fighters and in the control group. This was partially confirmed by the results of our study where cervical spine proprioception was related to balance control in taekwondo fighters when standing on the non-preferred leg, but not on the preferred leg. In the control group these correlations were present for all balance tasks. The second aim of our study was to assess whether balance deficits are more pronounced in posture when the head is rotated. Based on the results from our study this was not the case in taekwondo fighters but was present in the control group.

Taekwondo fighters seem to be able to maintain body sway control efficiently while their head is rotated and during the forward-facing balance task. Such adaptations could result from habituation to sport's specific demands that involve adaptations in whole body musculature. During single leg standing in taekwondo there is a constant need to preserve balance while perturbations are applied on the trunk via the kicking and standing leg. This demands adaptations in the whole kinetic chain and muscular synergies responsible for preserving whole body balance and posture during forward facing and head rotation balance tasks. The present was not seen in the control group where differences were found between forward facing and neck torsioned stances. Balance control during the latter was 
altered in the anterior-posterior direction for sway path and average amplitude. Changes in sway path and average amplitude during neck rotation could indicate different efficiency in maintaining balance control.

Although no differences were found in body sway between the legs in taekwondo fighters, there were differences present in the correlation to neck kinesthetic functions. Taekwondo fighters frequently place themselves in an asymmetrical posture. This can induce changes in neck muscle excitability and consequently alter neck muscle proprioception. This speculation is partially confirmed by the results of our study, where variable error in the Head-to-Neutral test from the non-preferred side correlated with average amplitude of body sway in anterior-posterior direction while standing on the non-preferred leg. The active repositioning test has been suggested to be dependent on the sensory drive, primarily from the muscle spindles [17]. Besides amplitude and velocity of the homologues muscle stretch, muscle spindle firing is dependent on the alpha-gamma coactivation controlled by the central nervous system [18]. Altered alpha-gamma coactivation could result in changed excitability of the muscle spindles and consequently their firing properties [18]. Due to possible asymmetrical changes in neck muscle tone, a proprioceptive mismatch could be present in taekwondo fighters, leading to an altered body representation in space $[7,19]$. In order to overcome such deficiencies, sensory-motor reweighing could take place, possibly changing the involvement of cervical proprioception in relation to body sway control as was observed in our study. Appropriate afferent input from cervical spine muscles could therefore significantly contribute to body sway feedback control [20].

Additionally, results of our study show that taekwondo fighters presented with relations between body sway while facing forward on the non-preferred leg and overshoot parameter during the Butterfly test. The overshoot parameter is a metric that describes the time that the head and neck spend ahead of the reference target during randomly changing directions of movement. Therefore, the largest proportion of overshoot can be caused by less efficient correction of movement direction. In this instance, it is necessary to accurately reverse the function of the muscles performing the initial impulse from agonists to antagonists in order to accurately decelerate and initiate changes in direction. According to Apker et al. [21], accurate movement control demands appropriate sensory feedback; therefore, proprioceptive deficits could result in inappropriate sensory motor coupling.

Further findings from our study imply that during the non-preferred neck torsion balance task, the correlation was present between most body sway parameters and the undershoot parameter of the Butterfly test. Undershoot parameter could indicate increased tonic muscular activity via increased muscle spindle firing which may mean that participants are unable to react accurately during unpredictable changes in the direction of movement. As taekwondo fighters must possess the ability to quickly change direction of movement while attacking and defending during a fight, such deficits could decrease sports performance.

Interestingly, no such correlations were observed between neck proprioception, neck movement control and postural balance while standing on their preferred leg in taekwondo fighters. This indicates that neck proprioception and movement control are not related to body sway control in their preferred guard. Such results can be interpreted as the body's attempt to decrease its reliance on neck proprioception. Factors such as fatigue [22], eccentric loading during hits to the head [23] or cervical soft tissue creep due to prolonged loading in the neck torsion posture [24] could lead to decreased reliance on cervical proprioception. Therefore, sensory reweighing must take place, where more reliable proprioceptive information is used for balance control [25]. Such adaptations are beneficial for taekwondo fighters as they enable more robust and adaptable in terms of body sway control, which is vital for maintaining tactical superiority over an opponent.

In order to preserve balance, the human body constantly corrects its posture, initiates changes in the center of mass position or moves the support surface while relying on different sensory information [26]. Based on the above observations, taekwondo fighters seem to involve cervical proprioception differently as compared to controls in relation to balance control. 
Postural balance is influenced by visual, proprioceptive and vestibular afferent input [25]. Visual and vestibular inputs become increasingly more important during dynamic movements of the head as seen during a taekwondo fight. In addition, afferent input from cervical muscles could induce adaptive plastic changes in vestibular dependent motion sensitiveness, not commonly taken into account when investigating balance deficits [27]. Therefore, more in-depth relations between different sensory inflow in relation to balance performance should be investigated.

Author Contributions: Conceptualization, Z.M.R.; methodology, M.V.; formal analysis, Z.M.R. and M.V.; investigation, Z.M.R.; writing-original draft preparation, Z.M.R.; writing-review and editing, Z.M.R. and M.V.; visualization, Z.M.R. and M.V.; supervision, Z.M.R. All authors have read and agreed to the published version of the manuscript.

Funding: This research received no external funding.

Conflicts of Interest: The authors declare no conflict of interest.

\section{References}

1. Kim, T.-W.; Lee, S.-C.; Kil, S.-K.; Kang, S.-C.; Lim, Y.-T.; Kim, K.-T.; Panday, S.B. Kicking modality during erratic-dynamic and static condition effects the muscular co-activation of attacker. J. Sports Sci. 2017, 35, 835-841. [CrossRef] [PubMed]

2. Yoo, S.; Park, S.-K.; Yoon, S.; Lim, H.S.; Ryu, J. Comparison of Proprioceptive Training and Muscular Strength Training to Improve Balance Ability of Taekwondo Poomsae Athletes: A Randomized Controlled Trials. J. Sports Sci. Med. 2018, 17, 445-454. [PubMed]

3. Kim, J.; Kwon, M.-S.; Yenuga, S.S.; Kwon, Y.-H. The effects of target distance on pivot hip, trunk, pelvis, and kicking leg kinematics in Taekwondo roundhouse kicks. Sports Biomech. 2010. [CrossRef] [PubMed]

4. Dai, B.; Layer, J.; Vertz, C.; Hinshaw, T.; Cook, R.; Li, Y.; Sha, Z. Baseline Assessments of Strength and Balance Performance and Bilateral Asymmetries in Collegiate Athletes. J. Strength Cond. Res. 2019, 33, 3015-3029. [CrossRef] [PubMed]

5. Sarabon, N.; Hirsch, K.; Majcen, Z. The acute effects of hip abductors fatigue on postural balance. Montenegrin J. Sports Sci. Med. 2016, 5, 5-9.

6. Boyd-Clark, L.C.; Briggs, C.A.; Galea, M.P. Muscle spindle distribution, morphology, and density in longus colli and multifidus muscles of the cervical spine. Spine 2002, 27, 694-701. [CrossRef] [PubMed]

7. Pettorossi, V.E.; Schieppati, M. Neck proprioception shapes body orientation and perception of motion. Front. Hum. Neurosci. 2014, 8, 895. [CrossRef]

8. Wannaprom, N.; Treleaven, J.; Jull, G.; Uthaikhup, S. Neck muscle vibration produces diverse responses in balance and gait speed between individuals with and without neck pain. Musculoskelet. Sci. Pract. 2018, 35, 25-29. [CrossRef]

9. Peterson, B.W. Current approaches and future directions to understanding control of head movement. Prog. Brain Res. 2004, 143, 369-381. [CrossRef]

10. Williams, K.; Tarmizi, A.; Treleaven, J. Use of neck torsion as a specific test of neck related postural instability. Musculoskelet. Sci. Pract. 2017, 29, 115-119. [CrossRef]

11. Winter, T.; Beck, H.; Walther, A.; Zwipp, H.; Rein, S. Influence of a proprioceptive training on functional ankle stability in young speed skaters-A prospective randomised study. J. Sports Sci. 2015, 33, 831-840. [CrossRef]

12. Kristjansson, E.; Oddsdottir, G.L. "The Fly": A new clinical assessment and treatment method for deficits of movement control in the cervical spine: Reliability and validity. Spine 2010, 35, E1298-E1305. [CrossRef] [PubMed]

13. Cohen, J. Statistical Power Analysis for the Behavioral Sciences; Elsevier: Amsterdam, The Netherlands, 1977; ISBN 978-0-12-179060-8.

14. Field, A. Discovering Statistics Using SPSS.; SAGE Publications: London, UK, 2009; ISBN 978-1-84787-907-3.

15. Ferreira, J.A.; Zwinderman, A.H. On the Benjamini-Hochberg method. Ann. Statist. 2006, 34, $1827-1849$. [CrossRef] 
16. Jamal, K.; Leplaideur, S.; Leblanche, F.; Moulinet Raillon, A.; Honoré, T.; Bonan, I. The effects of neck muscle vibration on postural orientation and spatial perception: A systematic review. Neurophysiol. Clin. 2020, 50, 227-267. [CrossRef] [PubMed]

17. Proske, U.; Gandevia, S.C. Kinesthetic Senses. Compr. Physiol. 2018, 8, 1157-1183.

18. Macefield, V.G.; Knellwolf, T.P. Functional properties of human muscle spindles. J. Neurophysiol. 2018, 120, 452-467. [CrossRef]

19. Schieppati, M.; Nardone, A.; Schmid, M. Neck muscle fatigue affects postural control in man. Neuroscience 2003, 121, 277-285. [CrossRef]

20. Panzer, V.P.; Bandinelli, S.; Hallett, M. Biomechanical assessment of quiet standing and changes associated with aging. Arch. Phys. Med. Rehabil. 1995, 76, 151-157. [CrossRef]

21. Apker, G.A.; Karimi, C.P.; Buneo, C.A. Contributions of vision and proprioception to arm movement planning in the vertical plane. Neurosci. Lett. 2011, 503, 186-190. [CrossRef]

22. Gandevia, S.C. Spinal and supraspinal factors in human muscle fatigue. Physiol. Rev. 2001, 81, 1725-1789. [CrossRef]

23. Proske, U. Exercise, fatigue and proprioception: A retrospective. Exp. Brain Res. 2019, 237, $2447-2459$. [CrossRef] [PubMed]

24. Schäfer, S.S. Regularity in the generation of discharge patterns by primary and secondary muscle spindle afferents, as recorded under a ramp-and-hold stretch. Exp. Brain Res. 1994, 102, 198-209. [CrossRef] [PubMed]

25. Peterka, R.J. Sensory integration for human balance control. Handb. of Clin. Neurol. 2018, 159, $27-42$. [CrossRef]

26. Rosker, J.; Markovic, G.; Sarabon, N. Effects of vertical center of mass redistribution on body sway parameters during quiet standing. Gait Posture 2011, 33, 452-456. [CrossRef]

27. Pettorossi, V.E.; Panichi, R.; Botti, F.M.; Biscarini, A.; Filippi, G.M.; Schieppati, M. Long-lasting effects of neck muscle vibration and contraction on self-motion perception of vestibular origin. Clin. Neurophysiol. 2015, 126, 1886-1900. [CrossRef]

Publisher's Note: MDPI stays neutral with regard to jurisdictional claims in published maps and institutional affiliations.

(C) 2020 by the authors. Licensee MDPI, Basel, Switzerland. This article is an open access article distributed under the terms and conditions of the Creative Commons Attribution (CC BY) license (http://creativecommons.org/licenses/by/4.0/). 